http://jmscr.igmpublication.org/home/ ISSN (e)-2347-176x ISSN (p) 2455-0450 crossref DOI: https://dx.doi.org/10.18535/jmscr/v9i2.15

\title{
Stress Myocardial Perfusion Scintigraphy in Triple Vessel Disease - Role of TID in Balanced ischemia
}

\author{
Authors \\ Tejonath Gadepalli*, Ranadheer Guptha Manthri, Santhi Bhushan Murari, \\ V V S Prabhakar Rao ${ }^{1}$ \\ ${ }^{1}$ Departments of Nuclear Medicine, Nizam`s Institute of Medical Sciences, Hyderabad, India \\ *Corresponding Author \\ Dr Tejonath G \\ Nuclear Medicine Department, Basavatarakam Indoamerican Cancer Hospital, Banjara Hills, No. 10, \\ Hyderabad - 500034, India
}

\begin{abstract}
Introduction: Stress myocardial perfusion scintigraphy has been one of the sensitive modalities to diagnose inducible ischemia. Theoretically one of the major pitfalls quoted is the lack of sensitivity in triple vessel disease due to balanced hypoperfusion. The aim of this investigation is to evaluate the efficacy of Gated Stress Myocardial Perfusion (MPI) SPECT in known patient of Triple Vessel Disease (TVD).

Materials and Methods: 34 patients of known TVD were subjected to single day Gated stress MPI using Tc 99 m sestamibi.

Results: Out of 34 patients perfusion abnormalities were detected in 31 patients (91.2\%). Twenty-three of them (67.6\%) had the defect in the territory of LAD, 11 (32\%) in LCX and 25 (73.5\%) in RCA. Transient Ischemic Dilatation (TID) was found in 24 patients (70 \%) associated with perfusion defect. One out of three patients with normal perfusion showed TID .False Negative rate for hemodynamically significant coronary artery stenosis if only perfusion defects are evaluated is $2.94 \%$. Management decision changed in 11 patients out of 34 patients (32.3\%). With increase in the percentage of coronary stenosis there is increase in probability of occurance of perusion defects on myocardial perfusion scan.

Conclusion: This study showed that combined assessment of perfusion patterns and gated SPECT help in risk stratification, TID can help in TVD with balance ischemia, However it is commonly associated with perfusion defects.

Keywords: Gated SPECT, Triple Vessel Disease, Transient Ischemic Dilatation.
\end{abstract}

\section{Introduction}

Stress myocardial perfusion scintigraphy has been one of the sensitive modalities to diagnose inducible ischemia. Theoretically one of the major pitfalls quoted is the lack of sensitivity in triple vessel disease due to balanced hypoperfusion.
The quantitative Gated Single Photon Emission Computed Tomography (SPECT) has improved the sensitivity in multiple coronary vessel disease significantly ${ }^{1}$. Frequently, stress myocardial perfusion imaging (MPI) is used to assess areas with the most ischemia to guide therapeutic intervention. It has been demonstrated that there is 
no difference in sensitivity or specificity for diagnosing coronary artery disease between three commercially available tracers ${ }^{2}$. In particular the number of reversible defects is directly proportional to the risk of significant events ${ }^{2}$

In this study we evaluated patients with known triple vessel disease using exercise or pharmacological (Dobutamine) stress for testing with quantitative gated SPECT MPI. The objective is to evaluate the incidence of Transient ischemic left ventricular dilatation (TID).

\section{Materials and Methods}

A prospective study, single institutional study was conducted in patients with angiographic evidence of Triple vessel coronary artery disease on conventional angiography within 3 months. we evaluated 34 adult patients. Patients were excluded from the study if they had undergone previous therapeutic coronary intervention, Pregnant or Lactating woman, Hypersensitive to radiopharmaceutical, Recent myocardial infarction (less than 72hrs), Uncontrolled hypertension \&Left main critical stenosis with rest symptoms. Thirty were male and four were female. The study was approved by hospital ethics committee. The patients underwent single day Stress (exercise/ Pharmacological) \& Rest MPI with 99mTc labelled methoxyisobutylisonitrile (MIBI).

\section{Stress Techniques}

Patients were given following instructions, 1)at least 4 hours fasting before the study, 2) They are advised to withhold $\beta$-blockers for 72 hours, calcium channel blockers for 24 hours and nitrates on the day of the test, 3)Patients are advised to fatty meal.

99mTC labelled sestamibi was injected at the peak stress (Exercise/Pharmacological) and at resting. Dosage 8-10mCi (296-370MBq) for stress study and $20-30 \mathrm{mCi}(740-1110 \mathrm{MBq})$ for rest study in a single day protocol.

\section{Positioning and Acquisition}

Imaging was performed on Siemens E Cam Gamma Camera (Dual Head). Patient comfortably positioned. Imaging was commenced $45 \mathrm{~min}$ after either stress or rest injection using single day protocol. Images were acquired with a Dual-head rotating large field of view camera, equipped with a low-energy, parallel-hole, all-purpose collimator. Both detectors at $90^{\circ}$ configuration circular orbit starting from $-45^{\circ}$ right anterior oblique to $+135^{\circ}$ left posterior oblique with the patient in the supine position with the energy discriminator set at peak energy of $140 \mathrm{keV}$ and a $20 \%$ symmetric window with analog zoom of 1.45, Projections $(n=32), 64 X 64$ matrixes, 32 views, step \& shoot, 25-30 seconds each frame. Circular and step and shoot method. Raw images were reconstructed by filtered back projection method. The tomographic data were used to reconstruct the left ventricle in the short-axis, horizontal long-axis and vertical long-axis orientations. Projection images were processed with a Butterworth filter having a cutoff frequency of 0.45 cycles per pixel and an order of 5.Cardiac SPECT images were reviewed independently by Nuclear Medicine Physician \& Cardiologist and compared with coronary angiography.

\section{Image Interpretation}

SPECT images were interpreted visually \&with adjunctive semi-quantitative analysis. Perfusion defects were defined as $30 \%$ or more decrease of myocardial tracer uptake in inferior wall and anterior wall of female and $20 \%$ or more decrease in other walls of left ventricle in stress, the anterior wall and septum were considered the territory of LAD; the inferior wall, the territory of RCA and the lateral wall, the territory of LCX. TID was measured by comparing ventricular cavity of stress with Rest SPECT images. TID values > 1.18 in men $\&>1.22$ in women were considered positive ${ }^{24}$.

\section{Coronary Angiography}

Coronary angiography was performed within 3 months of MPI study were considered \& visually interpreted by experienced cardiologists. All data has been divided into three categories: Borderline stenosis, moderate stenosis and severe stenosis based on coronary angiography interpretation. Borderline stenosis is classified as $60-70 \%$ 
stenosis, moderate cases included $\geq 70 \%$ \&< $90 \%$ stenosis, except stenosis $\geq 70 \%$ in the proximal left anterior descending artery (pLAD) or the left main (LM). Severe CAD is defined as $\geq 90 \%$ stenosis.

\section{Statistical Analysis}

Data was entered in Microsoft excel spread sheet version 2003. Data was categorized as presence on perfusion defect as $\mathrm{Y}$ and absence of disease as $\mathrm{N}$, and then expressed in actual numbers. Using 2 x 2 table sensitivity for detection of perfusion defect was estimated.

\section{Results}

In a total of 34 patients with Tc 99m-sestamibi myocardial perfusion scintigraphy and documented TVD in CAG, 30 patients were males and 4 females.

The inducible ischemia was found in all34 patients $(100 \%) .26$ ischemic defects in the territory of LAD (76.4\%), 11 in LCX (31\%) and 24 in RCA(70.5\%). Total 64ischemic defects were noted. Lateral wall was preserved in most of the cases $(67.6 \%)$. LAD territory was most commonly involved.

TID was associated with 36 ischemic perfusion defects $(56 \%)$, of which all 26ischemic defects in LAD territory (100\%) showed TID, 2 in LCX $(18 \%)$ and 8 in RCA territory (33.3\%).

With increase in the percentage of coronary stenosis there is increase in probability of occurance of perusion defects on myocardial perfusion scan.

However incidence of TID is higher with ischemia in LAD territory than in any other coronary territory. As the coronary stenosis increased the severity and extent of ischemia increased proportionally.

\section{Relation between TID ratio and perfusion defect}

The relation between the TID ratios and ischemic defect showed the LAD territory involvement had the biggest percentage of abnormal TID. There are significant increases of abnormal TID ratios with increase in severity of lesion on CAG.

Fig 1: Normal perfusion of tracer both a stress and rest

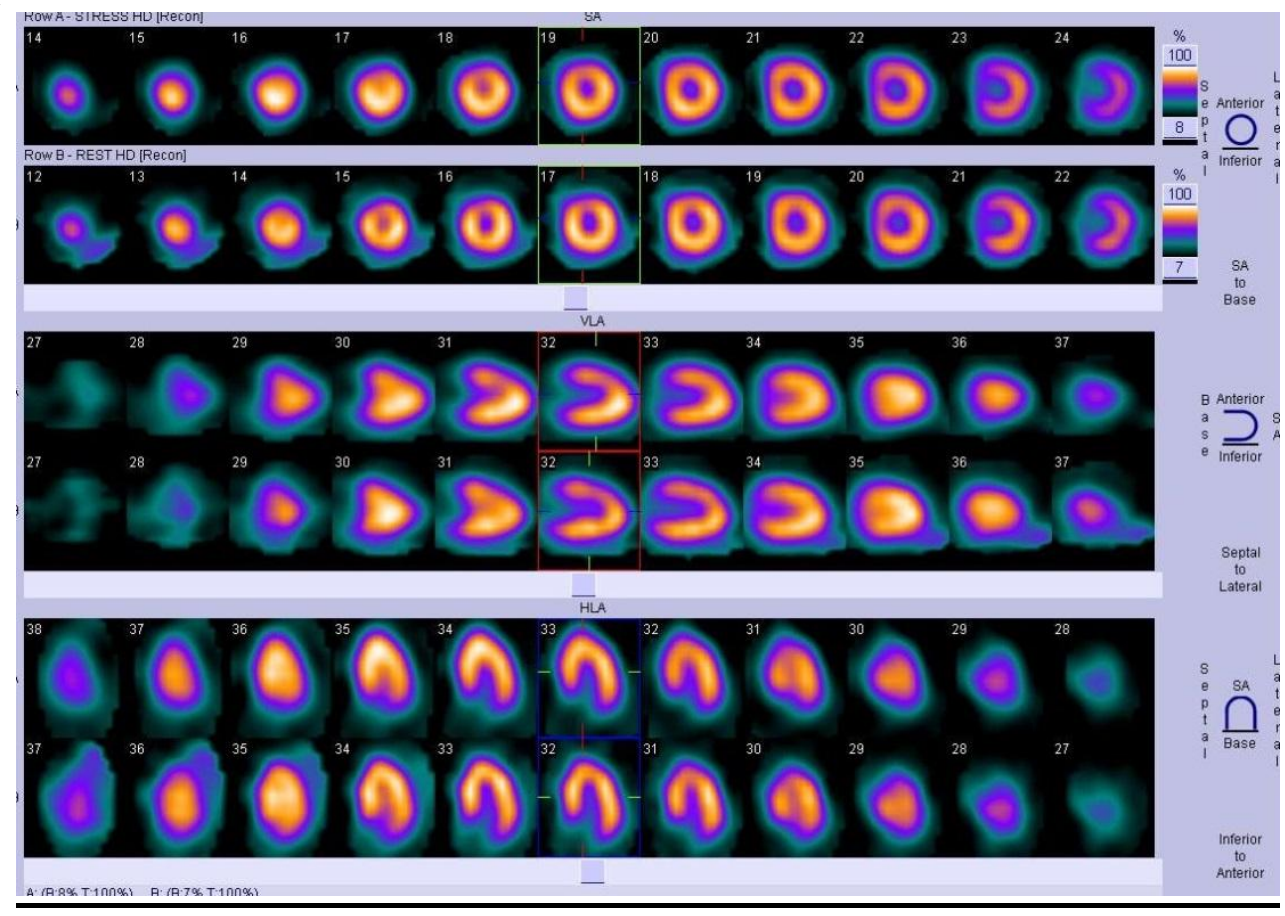




\section{JMSCR Vol||09||Issue||02||Page 96-103||February}

Fig 2: Stress inducible ischemia in LAD territory

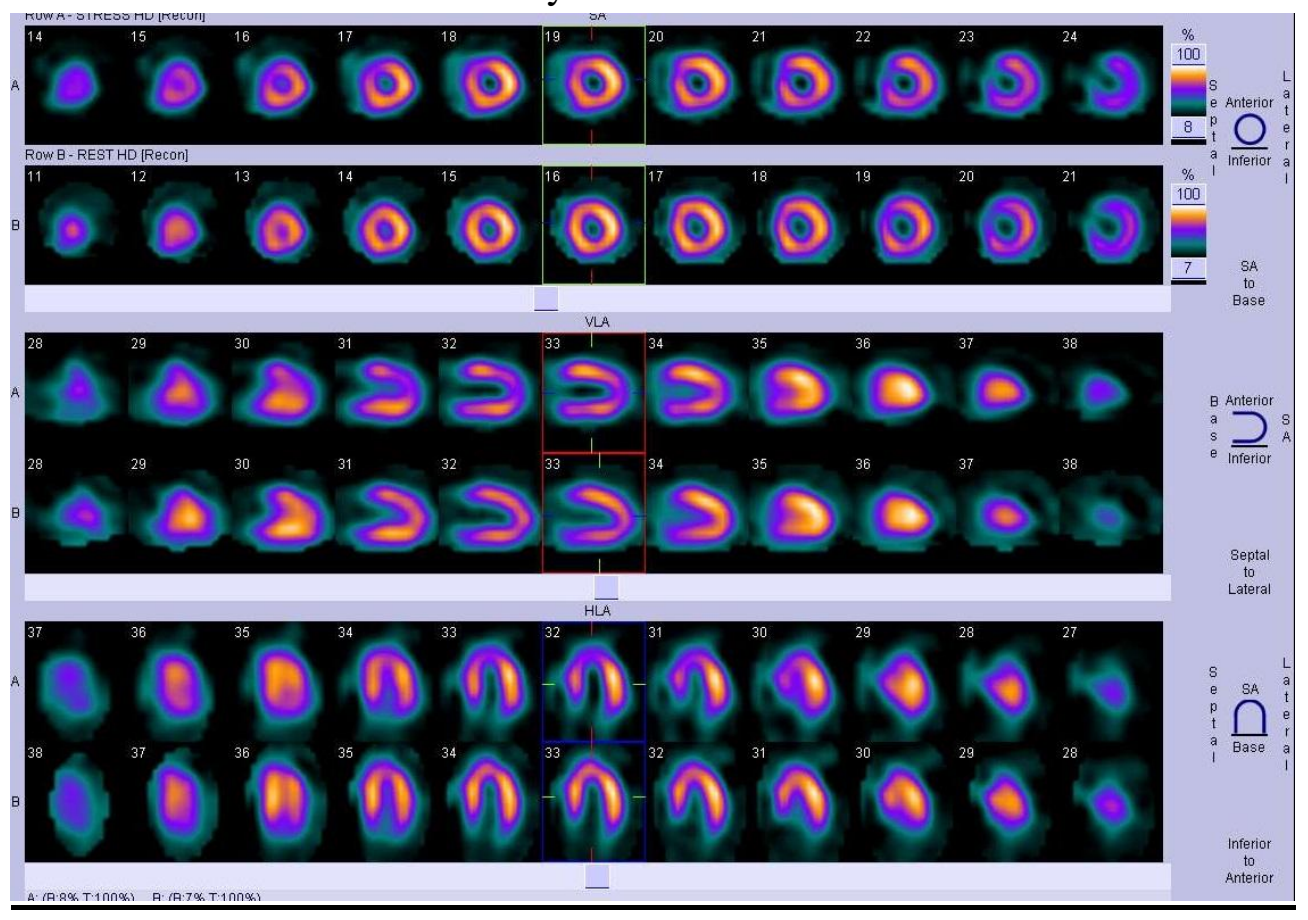

Fig 3: Stress inducible ischemia in LAD \& RCA territory

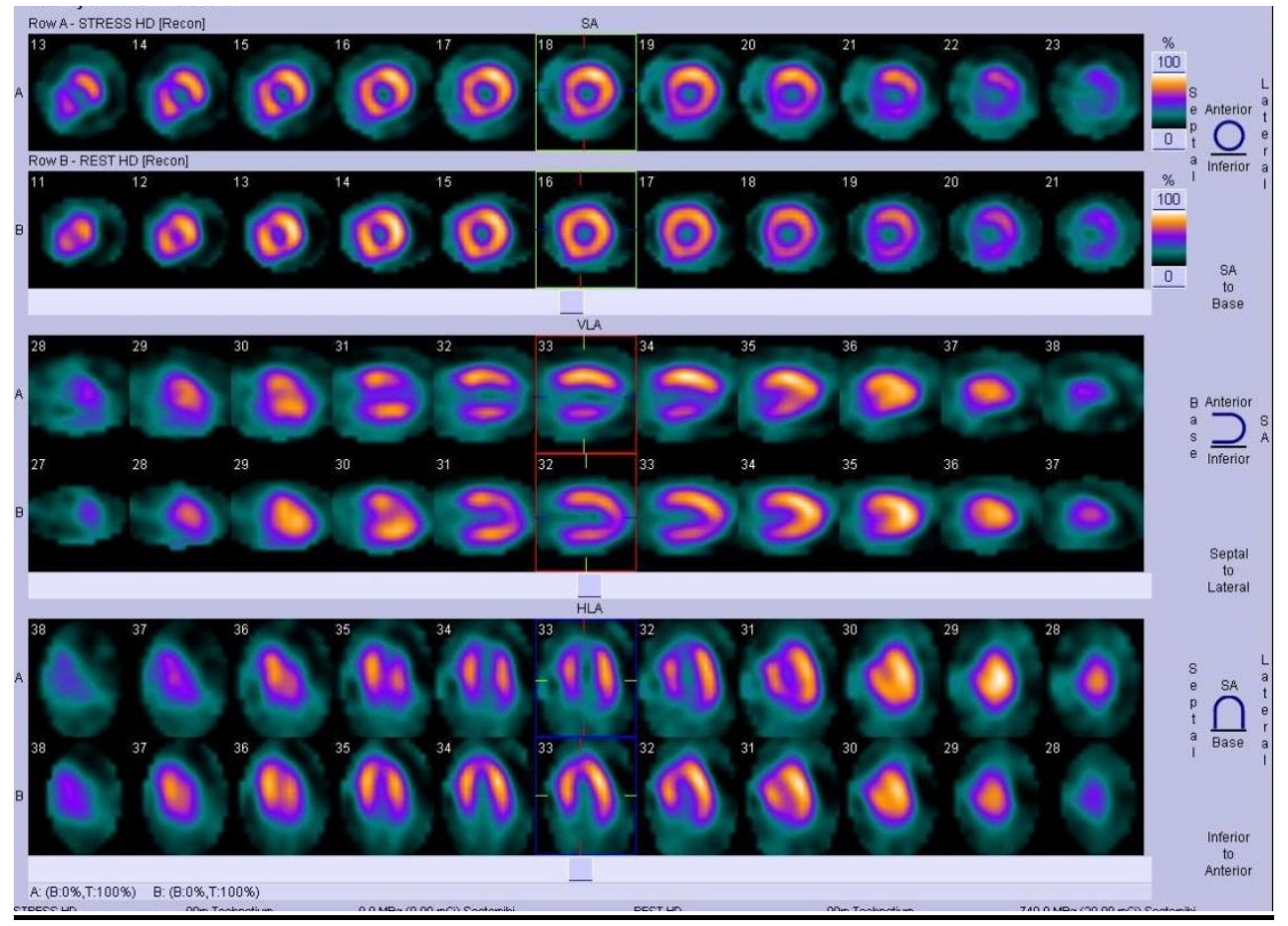




\section{JMSCR Vol||09||Issue||02||Page 96-103||February}

Fig 4: Stress inducible ischemia in LAD, LCX and RCA territory

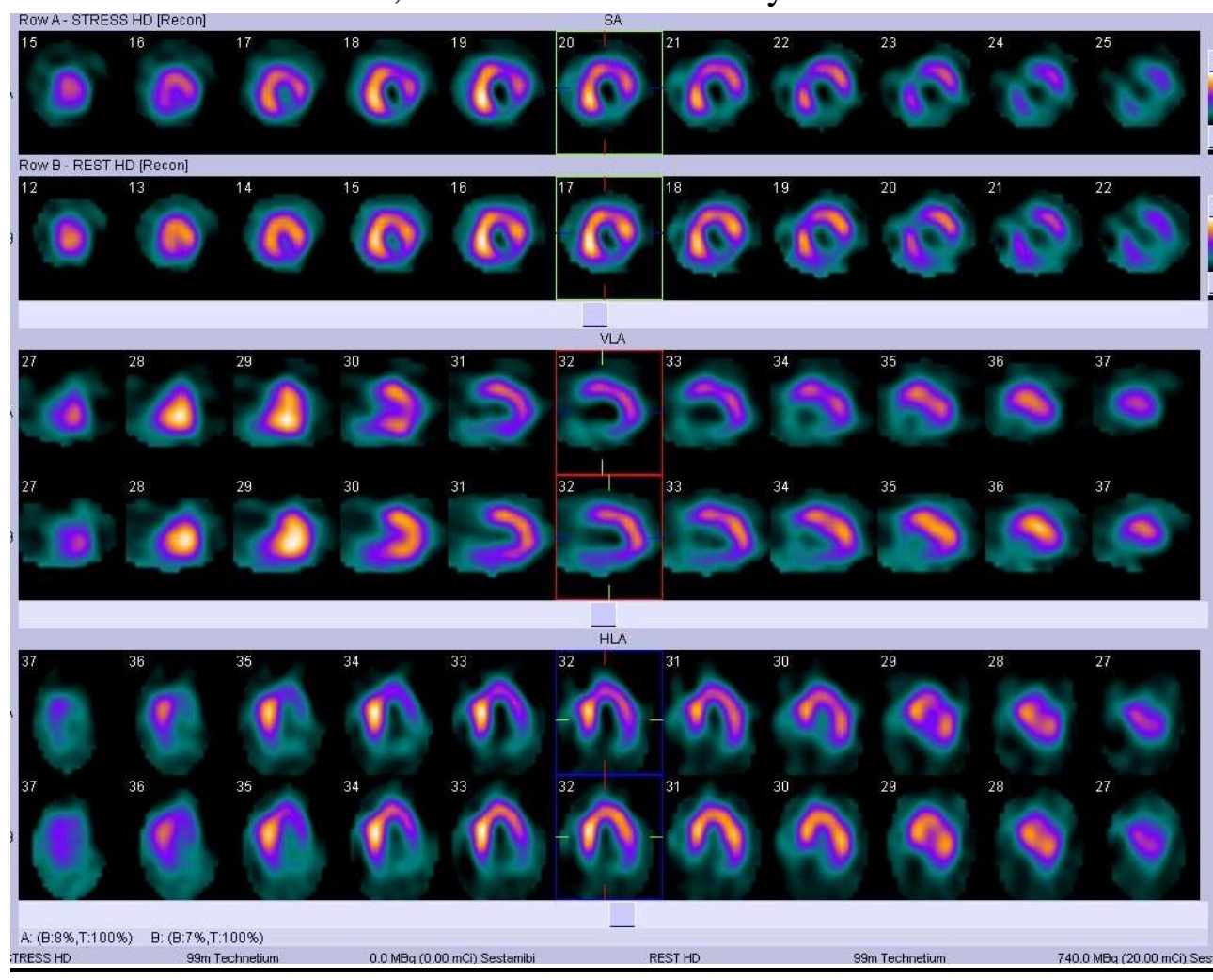

Fig 5: Normal perfusion with Transient Ischemic Dilatation and Stress induced dysfunction

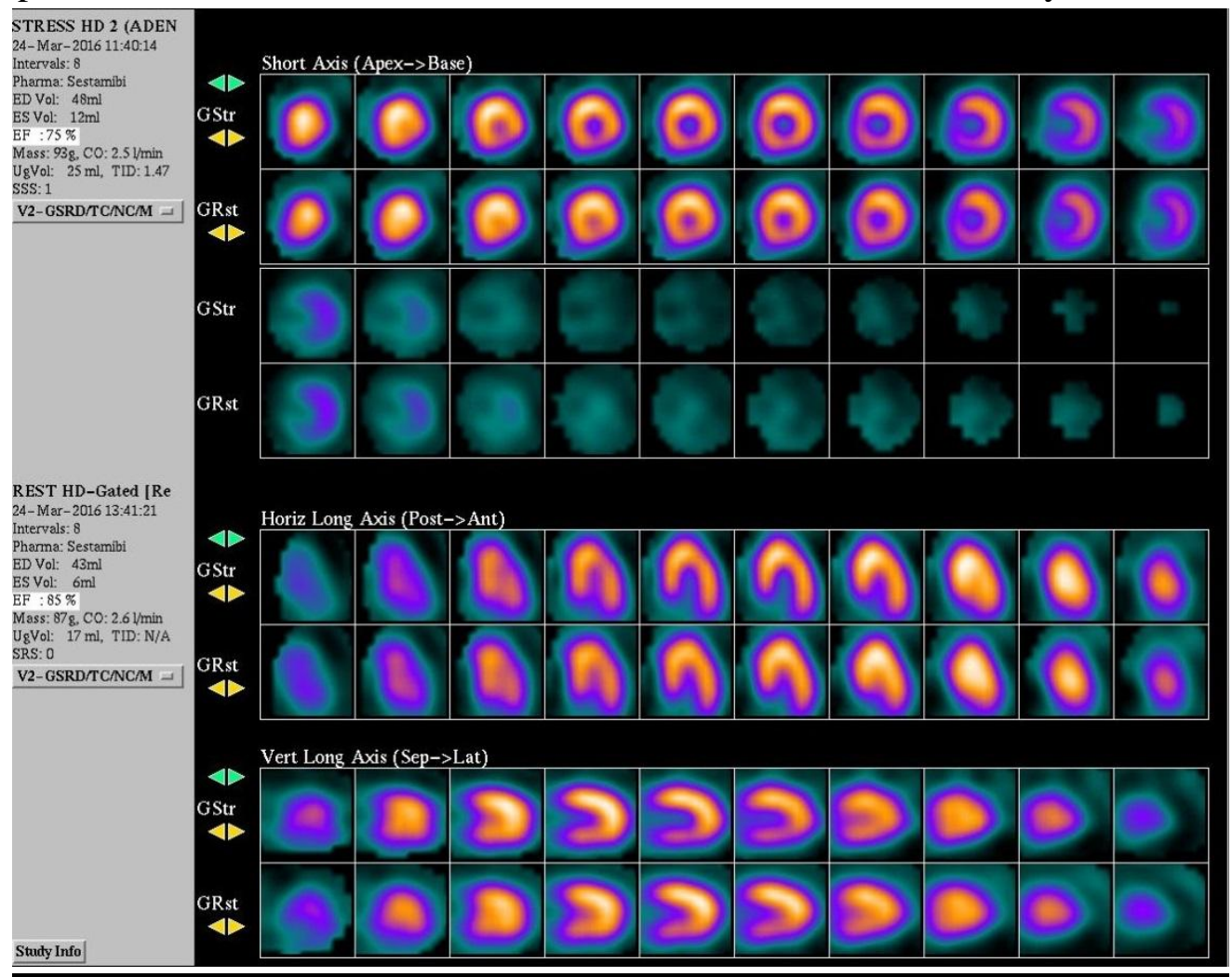

\section{Discussion}

There are 64 ischemic defects in 34 patients with TVD. LAD territory was most commonly involved with preserved lateral wall. Because the
Tc99m Sestamibi distribution reflects relative rather than absolute reduction in myocardial blood flow, areas with less hypoperfusion may appear relatively normal compared with the most severely 
hypoperfused segments. The normal distribution of post exercise $99 \mathrm{mTc}$ sestamibi uptake for men and women showed the highest activity on the lateral wall ${ }^{3}$. The lateral wall tends to have more counts than the other wall seven if it is abnormal. SPECT images are normalized to the region of the myocardium having the greatest count density. Therefore, in cases ofin homogeneity of absolute $99 \mathrm{mTc}$ sestamibi uptake, it is quite possible that the best perfused region is in fact preserved in the spatially relative perfusion defect analysis.

In our study, the overall sensitivity of SPECT myocardial perfusion images for inducible ischemia in triple vessel disease was $100 \%$ (34/34), which is more than the results of several reports, the mean sensitivity increases for patients with TVD ${ }^{4-7}$.

Previous investigation has shown that the magnitude of ischemia has an exponential relationship to the occurrence of subsequent cardiac event ${ }^{8}$. Iskandrian et al. conducted a 2year follow-up of 449 patients following the performance of $201 \mathrm{Tl}$ myocardial perfusion scintigraphy 9 .

Brown and Rowen reported only two cardiac events during a mean follow-up of 24 months among 75 consecutive patients who manifested a normal exercise myocardial perfusion scintigraphy study in conjunction with angiographic evidence of significant $\mathrm{CAD}$, yielding an annualized rate of cardiac event of only $0.7 \%$ /year ${ }^{10}$. The presence of a normal scintigraphic study confers a very benign prognosis, even in patients with strongly positive exercise electrocardiograms or angiographically significant $\mathrm{CAD}^{4,10-12}$. However, the numbers of this group might be underestimated because the widespread clinical acceptance of preferential selection of patients for CAG based on a positive myocardial perfusion imaging study result, and the refraining from catheterization of patients with a negative test result.

TID generally markers of both extensive and severe $\mathrm{CAD}^{13,14-18,19-21}$. Hansen et al suggested that TID represent pathophysiologic responses to exercise-induced ischemia $^{22}$. TID represents transient subendocardial ischemia, in our study, TID was associated with 36 ischemic perfusion defects $(56 \%)$, of which all 26 ischemic defects in LAD territory (100\%) showed TID, 2 in LCX $(18 \%)$ and 8 in RCA territory (33.3\%). In this study, we found the presence of TID is most frequently associated with LAD territory ischemia than in any other coronary territory because of hemodynamic significance of LAD. The occurrence TID increases with increase in severity of stenosis on CAG.

\section{Conclusion}

This study showed that combined assessment of perfusion patterns and gated SPECT help in risk stratification. We have evaluated performance of TID for the detection of severe CAD. The results suggest that the TID ratios will provide incremental diagnostic \& prognostic information to standard MPI study in semi quantitative analysis for the evaluation of patients with multi vessel CAD.

\section{Bibliography}

1. Maddahi J, Rodrigues E, Kiat H, Van Train KF, Berman DS: Detection and evaluation of coronary artery disease by thallium-201 myocardial perfusion scintigraphy. In: DePuey EG, Berman DS, Garcia EV (eds). Cardiac SPECT Imaging. New York: Raven Press, 1995, pp 103-20

2. Kapur A, Latus K, Davies G et al 2002, Eur J Nucl Med 29: 1608-1616

3. DePasquale EE, Nody AC, De Puey EG, et al. Quantitative rotational thallium-201 tomography for identifying and localizing coronary artery disease. Circulation 1988;77:316-327.

4. Maddahi J, Van Train K, Prigent F, et al. Quantitative single photon emission computed thallium-201 tomography for detection and localization of coronary artery disease, optimization and 
prospective validation of a new technique. J Am Coll Cardiol 1989;14:1689-1699.

5. Iskandrian AS, Heo J, Kong B, Lyons E. Effect of exercise level on the ability of thallium-201 tomographic imaging in detecting coronary artery disease: analysis of 461 patients. J Am Coll Cardiol 1989;14:1477-1486.

6. Mahmarian JJ, Boyce TM, Goldberg RK, et al. Quantitative exercise thallium-201 single photon emission computed tomography for the enhanced diagnosis of ischemic heart disease. J Am Coll Cardiol 1990;15:318- 329.

7. Van Train KF, Maddahi J, Berman DS, et al. Quantitative analysis of tomographic stress thallium-201 myocardial scintigrams: a multicenter trial. J Nucl Med 1990;31:1168-1179.

8. Ladenheim ML, Pollock BH, Rozanski A, et al. Extent and severity of myocardial hypoperfusion as predictors of prognosis in patients with suspected coronary artery disease. J Am CollCardiol 1986;7:464471. Ann Nucl Med Sci2002;15:175-182 Vol. 15 No. 4 December 2002180 Scintigraphic findings in TVD

9. Iskandrian AS, Heo J, Decoskey D, Askenase A, Segal BL. Use of exercise thallium-201 imaging for risk stratification of elderly patients with coronary artery disease. Am J Cardiol 1988;61:269-272.

10. Brown KA, Rowen M. Prognostic value of a normal exercise myocardial perfusion imaging study in patients with angiographically significant coronary artery disease. Am J Cardiol 1993;71:865867.

11. Abdel Fattah A, Kamal AM, Pancholy S, et al. Prognostic implications of normal exercise tomographic thallium images in patients with angiographic evidence of significant coronary artery disease. Am J Cardiol 1994;74:769-771.
12. Pavin D, Delonca J, Siegenthaler M, Doat M, Rutishauser W, Righetti A. Long-term (10 years) prognostic value of a normal thallium-201 myocardial exercise scintigraphy in patients with coronary artery disease documented byangiography. Eur Heart J 1997;18:69-77.

13. Tamaki N, Yonekura Y, Mukai T, et al. Stress thallium- 201 transaxial emission computed tomography: quantitative versus qualitative analysis for evaluation of coronary artery disease. J Am CollCardiol 1984;4:1213-1221.

14. Fintel DJ, Links JM, Brinker JA, et al. Improved diagnostic performance of exercise thallium-201 single photon emission computed tomography over planar imaging in the diagnosis of coronary artery disease: a receiver operating characteristic analysis. J Am CollCardiol 1989;13:600-612.

15. Weiss AT, Berman DS, Lew AS, et al. Transient ischemic dilation of the left ventricle on stress thallium-201 scintigraphy: a marker of severe and extensive coronary artery disease. J Am Coll Cardiol 1987;9:752-759.

16. Gibson RS, Watson DD, Carabello BA, Holt ND, Beller GA. Clinical implications of increased lung uptake of thallium-201 during exercise scintigraphy 2 weeks after myocardial infarction. Am J Cardiol 1982;49:1586-1593.

17. Bingham JB, McKusick KA, Strauss HW, Boucher CA, Pohost GM. Influence of coronary artery disease on pulmonary uptake of thallium-201. Am J Cardiol 1980;46:821-826.

18. Kushner FG, Okada RD, Kirshenbaum HD, Boucher CA, Strauss HW, Pohost GM. Lung thallium-201 uptake after stress testing in patients with coronary artery disease. Circulation 1981;63:341-347.

19. Levy R, Rozanski A, Berman DS, et al. Analysis of the degree of pulmonary 
thallium washout after exercise in patients with coronary artery disease. J Am Coll Cardiol 1983;2:719-728.

20. Maddahi J, Berman DS. Reverse redistribution of thallium- 201. J Nucl Med 1995;36:1019-1021.

21. Chouraqui P, Rodrigues EA, Berman DS, Maddahi J. Significance of dipyridamoleinduced transient dilation of the left ventricle during thallium-201 scintigraphy in suspected coronary artery disease. Am J Cardiol 1990;66:689-694.

22. Mazzanti M, Germano G, Kiat H, et al. Identification of severe and extensive coronary artery disease by automatic measurement of transient ischemic dilation of the left ventricle in dual-isotope myocardial perfusion SPECT. J Am Coll Cardiol 1996;27:1612-1620.

23. Kaul S, Finkelstein DM, Homma S, Leavitt M, Okada RD, Boucher CA. Superiority of quantitative exercise thallium-201 variables in determining long-term prognosis in ambulatory patients with chest pain: a comparison with cardiac catheterization. J Am Coll Cardiol 1988;12:25-34.

24. Hansen CL, Sangrigoli R, Nkadi E, Kramer M. Comparison of pulmonary uptake with transient cavity dilation after exercise thallium-201 perfusion imaging. JAmCollCardiol 1999;33:1323-1327.

25. Nuclear Medicine Communications: May 2003 - Volume 24 - Issue 5 - pp 543-549.

26. Halligan WT, Morris PB, Schoepf UJ, et al. Transient Ischemic Dilation of the Left Ventricle on SPECT: Correlation With Findings at Coronary CT Angiography. J Nucl Med. 2014;55:917-922. 